\title{
Flat Nonholonomic Matching
}

\author{
Dmitry V. Zenkov ${ }^{1}$ \\ Department of Mathematics \\ North Carolina State University \\ Raleigh, NC 27695 \\ dvzenkov@unity.ncsu.edu
}

\author{
Anthony M. Bloch ${ }^{2}$ \\ Department of Mathematics \\ University of Michigan \\ Ann Arbor, MI 48109 \\ abloch@math. .sa.umich.edu
}

\author{
Jerrold E. Marsden ${ }^{3}$ \\ Control and Dynamical Systems \\ California Institute of Technology 107-81 \\ Pasadena, CA 91125 \\ marsden@cds.caltech.edu
}

\begin{abstract}
In this paper we extend the matching technique to a class of nonholonomic systems with symmetries. Assuming that the momentum equation defines an integrable distribution, we introduce a family of reduced systems. The method of controlled Lagrangians is then applied to these systems resulting in a smooth stabilizing controller.
\end{abstract}

\section{Introduction}

In this paper we consider the problem of stabilization of relative equilibria of underactuated nonholonomic systems with symmetry by the method of controlled Lagrangians. The method of controlled Lagrangians for holonomic systems originated in Bloch, Leonard, and Marsden [4] and was then developed in Auckly [1], Bloch, Leonard, and Marsden [5, 6, 7], Bloch, Chang, Leonard, and Marsden [8], and Hamberg [11, 12]. A similar approach for Hamiltonian controlled systems was introduced and further studied in $[2,14,15,16]$.

According to this method, the original controlled system is represented as a new, uncontrolled Lagrangian system for a suitable controlled Lagrangian. The energy associated with this controlled Lagrangian is designed to be positive or negative definite at the (relative) equilibrium to be stabilized. The time invariant feedback control law is obtained from the equivalence

\footnotetext{
${ }^{1}$ Research partially supported by a University of Michigan Rackham Fellowship and an NSF group infrastructure grant at the University of Michigan

${ }^{2}$ Research partially supported by NSF grants DMS-9803181 and DMS-0103895, AFOSR grant F49620-96-1-0100, and an NSF group infrastructure grant at the University of Michigan

${ }^{3}$ Research partially supported by AFOSR grant F49620-95-1-
}

requirement for the new and old systems. If asymptotic stabilization is desired, the dissipation emulating terms are added to the control input.

Our goal is to extend this approach to systems subject to nonholonomic constraints. Some results in this direction were obtained in Zenkov, Bloch, and Marsden [18]. An algorithm leading to the nonholonomic version of the method of controlled Lagrangians was suggested for stabilizing the steady state motions of underactuated systems with two internal degrees of freedom. However, the practical implementation of this algorithm can lead to substantial difficulties, which have their roots in the complicated structure of the local invariant manifolds of the system in the neighborhood of the relative equilibrium subject to stabilization.

In the present paper we consider a somewhat restricted class of nonholonomic systems. We assume that the momentum equation is in the form of an integrable transport equation, which results in a simple structure of the global invariant manifolds of the system. We then apply the matching technique to the Lagrangian systems on these invariant manifolds.

To illustrate the theory, we consider the problem of stabilization of slow upright steady state motions of the unicycle along a straight line. It is well known that this motion becomes unstable if the forward speed of the unicycle is smaller than some critical value. We implement the controller by means of a balanced rotor placed on the top of a rod attached to the center of the unicycle. We then find the explicit stabilizing feedback control input. 


\section{Overview of Nonholonomic Dynamics}

In this section we give a brief exposition of nonholonomic dynamics. We refer the reader to Bloch, Krishnaprasad, Marsden, and Murray [3] and Zenkov, Bloch, and Marsden [17] for a complete exposition.

Symmetries. Suppose we are given a nonholonomic system specified by the Lagrangian $L: T Q \rightarrow \mathbb{R}$ and a (nonintegrable) constraint distribution $\mathcal{D}$. The Lagrangian has the form of kinetic minus potential energy. As usual, the kinetic energy is defined by a Riemannian metric on the manifold $Q$. We can then look for a group $G$ that acts (freely and properly) on the configuration space $Q$. It induces an action on the tangent bundle $T Q$ and so it makes sense to ask that the Lagrangian $L$ and the distribution $\mathcal{D}$ are invariant. If these properties hold, we say that $G$ is a symmetry group. In many examples the symmetry group will be evident. For instance, for the system considered below, the unicycle with rotor, the symmetry group is $S O(2) \times S E(2)$. The manifold $Q / G$ is called the shape space. The phase coordinates of a system with symmetry naturally form three groups: the shape, the momentum, and the group variables. The dynamics of the group variables is governed by the reconstruction equation. Since the system is $G$-invariant, this reconstruction equation decouples from the full system of equations of motion. Remaining equations are specified in the next paragraph.

Reduced Equations. These equations govern the evolution of the shape and momentum variables of the system. They are derived in Bloch, Krishnaprasad, Marsden, and Murray [3]. In the present paper we consider a class of nonholonomic systems that satisfies the following assumptions:

1. The curvature of the nonholonomic connection is zero.

2. The controls affect some of the shape variables of the system.

3. The momentum equation is in the form of the parallel transport equation

The Routhian of the system equals

$$
\mathcal{R}(r, \dot{r}, p)=\frac{1}{2} g_{\alpha \beta}(r) \dot{r}^{\alpha} \dot{r}^{\beta}-U(r, p),
$$

where the first term represents the shape metric and the second term, called the amended potential, is defined by

$$
U(r, p)=\frac{1}{2} I^{a b}(r) p_{a} p_{b}+V(r) .
$$

Here and below, $I^{a b}(r)$ are the components of the inverse locked inertia tensor and $V(r)$ is the potential energy of the system. As usual, the shape configuration variables and the nonholonomic momentum are denoted by $r$ and $p$, respectively. The reduced equations of a system satisfying the assumptions 1-3 become

$$
\begin{aligned}
\frac{d}{d t} \frac{\partial \mathcal{R}}{\partial \dot{r}^{\alpha^{\prime}}} & =\nabla_{\alpha^{\prime}} \mathcal{R} \\
\frac{d}{d t} \frac{\partial \mathcal{R}}{\partial \dot{r}^{\alpha^{\prime \prime}}} & =\nabla_{\alpha^{\prime \prime}} \mathcal{R}+u_{\alpha^{\prime \prime}} \\
\dot{p}_{a} & =\mathcal{D}_{a \alpha}^{b} p_{b} \dot{r}^{\alpha} .
\end{aligned}
$$

In the above, $r^{\alpha^{\prime}}$ and $r^{\alpha^{\prime \prime}}$ are the unactuated and actuated shape variables, respectively, and $u_{\alpha^{\prime \prime}}$ are the control inputs. The operators $\nabla_{\alpha}$ are defined by

$$
\nabla_{\alpha}=\frac{\partial}{\partial r^{\alpha}}+\mathcal{D}_{a \alpha}^{b} p_{b} \frac{\partial}{\partial p_{a}}
$$

See $[3,17]$ for details. The equilibria of these equations represent the steady state motions of the original system.

In the present paper we require, as a part of the controller design, that the actuated variables $r^{\alpha^{\prime \prime}}$ are cyclic, in which case the reduced equations are written as

$$
\begin{aligned}
\frac{d}{d t} \frac{\partial \mathcal{R}}{\partial \dot{r}^{\alpha^{\prime}}} & =\nabla_{\alpha^{\prime}} \mathcal{R}, \\
\frac{d}{d t} \frac{\partial \mathcal{R}}{\partial \dot{r}^{\alpha^{\prime \prime}}} & =u_{\alpha^{\prime \prime}}, \\
\dot{p}_{a} & =\mathcal{D}_{a \alpha^{\prime}}^{b} p_{b} \dot{r}^{\alpha^{\prime}} .
\end{aligned}
$$

Note that our definition of cyclic variables allows only non-cyclic shape velocities to occur in the momentum equation (3). Hence, the internal position of the controller has no impact on its performance.

Elimination of the Momentum Variables. Since the momentum equation is in the form of a parallel transport equation, it defines a distribution

$$
d p_{a}=\mathcal{D}_{a \alpha^{\prime}}^{c} p_{c} d r^{\alpha^{\prime}}
$$

We assume in this paper that the curvature of this distribution vanishes (hence the name flat in the title of the paper). This defines the global invariant manifolds $Q_{c}$ of (1)-(3):

$$
p_{a}=\mathcal{P}_{a}\left(r^{\alpha^{\prime}}, c_{b}\right), \quad c_{b}=\text { const. }
$$

Each of these invariant manifolds is diffeomorphic to the tangent bundle $T(Q / G)$ of the original system's shape space. The dynamics on these invariant manifolds is governed by the equations

$$
\frac{d}{d t} \frac{\partial \mathcal{L}_{\mathrm{c}}}{\partial \dot{r}^{\alpha^{\prime}}}=\frac{\partial \mathcal{L}_{\mathrm{c}}}{\partial r^{\alpha^{\prime}}}, \quad \frac{d}{d t} \frac{\partial \mathcal{L}_{\mathrm{c}}}{\partial \dot{r}^{\alpha^{\prime \prime}}}=u_{\alpha^{\prime \prime}}
$$

where

$$
\mathcal{L}_{c}\left(r^{\alpha^{\prime}}, \dot{r}^{\alpha}\right)=\mathcal{R}\left(r^{\alpha^{\prime}}, \dot{r}^{\alpha}, \mathcal{P}_{a}\left(r^{\alpha^{\prime}}, c_{b}\right)\right)
$$


We thus obtain a family of the underactuated controlled Lagrangian systems on $Q / G$. The Lagrangians $\mathcal{L}_{c}$ of these systems are represented by the formula

$$
\mathcal{L}_{c}=\frac{1}{2} g_{\alpha \beta} \dot{r}^{\alpha} \dot{r}^{\beta}-U\left(r^{\alpha^{\prime}}, \mathcal{P}\left(r^{\alpha^{\prime}}, c\right)\right)
$$

The structure of the Lagrangians $\mathcal{L}_{c}$.reveals the independence of the kinetic energy $\mathcal{K}_{c}$ on the vector parameter $c$.

\section{Matching and Controlled Lagrangians}

In this section we briefly discuss the matching technique. We refer the reader to $[1,4-8,11,12]$, for a detailed exposition.

Lagrangian Matching. Consider a mechanical system specified by the Lagrangian $L=K-V$. The kinetic energy $K$ is given by the Riemannian metric $g_{i j}$ on the configuration manifold $Q$. The potential energy $V(q)$ has a critical point at $q_{0}$. Assuming that the equilibrium $q_{0}$ is unstable, we would like to find the feedback control inputs that stabilize this equilibrium. This problem becomes interesting and nontrivial if the system is underactuated, i.e., the number of the control inputs is smaller than $\operatorname{dim} Q$.

Denote the unactuated and actuated variables by $x=$ $\left(x^{1}, \ldots, x^{m}\right)$ and $y=\left(y^{1}, \ldots, y^{n}\right)$, respectively. The controlled dynamics is governed by the equations

$$
\frac{d}{d t} \frac{\partial L}{\partial \dot{x}}=\frac{\partial L}{\partial x}, \quad \frac{d}{d t} \frac{\partial L}{\partial \dot{y}}=\frac{\partial L}{\partial y}+u,
$$

where $u=\left(u_{1}, \ldots, u_{n}\right)$ represents the control inputs.

According to the method of controlled Lagrangians, one introduces a new function $\widetilde{L}=\widetilde{K}-\widetilde{V}$ and considers the system

$$
\frac{d}{d t} \frac{\partial \widetilde{L}}{\partial \dot{x}}=\frac{\partial \widetilde{L}}{\partial x}, \quad \frac{d}{d t} \frac{\partial \widetilde{L}}{\partial \dot{y}}=\frac{\partial \widetilde{L}}{\partial y} .
$$

One then requires that the vector fields defined by $(8)$ and (9) are identical. This determines the feedback control inputs $u$. If in addition $\widetilde{K}+\widetilde{V}$ has a minimum (maximum) at $\left(q_{0}, 0\right)$, the equilibrium $q_{0}$ of the closed loop system (8) is neutrally stable.

The equivalence of the systems (8) and (9) imposes certain restrictions on $L$ and $\widetilde{L}$ represented by a system of nonlinear partial differential equations called the matching conditions. See $[1,11,12]$ for details and discussion of solvability of this system.

In [4-7] the technique of controlled Lagrangians is developed for stabilization of relative equilibria of mechanical systems with cyclic symmetries. The algorithm suggested in this series of papers allows one to find the explicit solution of the system of partial differential equations that represents the matching conditions for a special class of Lagrangians. The controlled Lagrangian is constructed in the form of $\widetilde{L}=\widetilde{K}-V$, where

$$
\widetilde{K}=K(x, \dot{x}, \dot{y}+k(x) \dot{x})+K_{\sigma}(x, k(x) \dot{x}),
$$

$K$ and $V$ are the original kinetic and potential energies, $K_{\sigma}$ is the quadratic form in $\dot{y}$ (with coefficients depending on $x$ ), and $k(x)$ is a vector-valued linear form in $\dot{x}$. The coefficients of the forms $k$ and $K_{\sigma}$ are denoted by $k_{\alpha^{\prime}}^{\alpha^{\prime \prime}}$ and $\sigma_{\alpha^{\prime \prime} \beta^{\prime \prime}}$, respectively. According to [7], one can match equations (8) and (9) if the following matching conditions are satisfied:

$$
\begin{aligned}
k_{\alpha^{\prime}}^{\alpha^{\prime \prime}} & =-\sigma^{\alpha^{\prime \prime} \beta^{\prime \prime}} g_{\alpha^{\prime} \beta^{\prime \prime}}, \\
\sigma^{\alpha^{\prime \prime} \beta^{\prime \prime}}\left(\sigma_{\beta^{\prime \prime} \gamma^{\prime \prime}, \alpha^{\prime}}+g_{\beta^{\prime \prime} \gamma^{\prime \prime}, \alpha^{\prime}}\right) & =2 g^{\alpha^{\prime \prime} \beta^{\prime \prime}} g_{\beta^{\prime \prime} \gamma^{\prime \prime}, \alpha^{\prime}} \\
k_{\alpha^{\prime}, \beta^{\prime}}^{\alpha^{\prime \prime}}-k_{\beta^{\prime}, \alpha^{\prime}}^{\alpha^{\prime \prime}} & =g^{\alpha^{\prime \prime} \beta^{\prime \prime}} g_{\beta^{\prime \prime} \gamma^{\prime \prime}, \alpha^{\prime}} k_{\beta^{\prime}}^{\gamma^{\prime \prime}}
\end{aligned}
$$

There is a certain freedom in choosing $k(x)$ and $K_{\sigma}$, which allows one to accomplish the problem of stabilization of relative equilibria. We omit here the details (see [7]), but note that this approach is applicable to systems whose kinetic energy is a quadratic form with constant coefficients. After $\widetilde{L}$ is constructed, the control inputs can be evaluated explicitly.

Application to Nonholonomic Systems with Integrable Momentum Equation. We now apply the matching procedure to the family of systems (6). Recall that the flatness of the distribution (4) is essential for defining this family. Each of the matching procedures outlined above starts from kinetic shaping, i.e., from constructing the controlled kinetic energy form. Since the kinetic energy of each systern in (6) is $c$-independent, the kinetic shaping can be accomplished for the whole family of Lagrangians $\mathcal{L}_{c}$ at once. In particular, if kinetic shaping is sufficient for stabilization, the control law obtained this way is represented by the same formula for all of the systems in (6). If potential shaping, i.e., the change of the potential energy, is required for stabilization, it is performed for each of the systems in (6) separately.

Assuming that potential shaping is not needed, we now discuss the procedure of obtaining the controlled Routhian from the family of the controlled Lagrangians (7). First, apply (10) to the family (7) and obtain the family of controlled Lagrangians $\widetilde{\mathcal{L}}_{c}=\widetilde{K}-$ $U\left(r^{\alpha^{\prime}}, \mathcal{P}\left(r^{\alpha^{\prime}}, c\right)\right)$. Then define the controlled Routhian by

$$
\widetilde{\mathcal{R}}=\widetilde{K}-U\left(r^{\prime}, p\right) .
$$

Theorem 1. Assume that the distribution (4) is flat and the matching conditions (11)-(13) are satisfied. Then equations (1)-(3) are equivalent to the equations of motion of a nonholonomic system associated with the 
controlled Routhian (14) and the original constrained distribution $\mathcal{D}$ :

$$
\begin{aligned}
\frac{d}{d t} \frac{\partial \widetilde{\mathcal{R}}}{\partial \dot{r}^{\alpha^{\prime}}} & =\nabla_{\alpha^{\prime}} \widetilde{\mathcal{R}} \\
\frac{d}{d t} \frac{\partial \widetilde{\mathcal{R}}}{\partial \dot{r}^{\alpha^{\prime \prime}}} & =0 \\
\dot{p}_{a} & =\mathcal{D}_{a \alpha^{\prime}}^{b} p_{b} \dot{r}^{\alpha^{\prime}} .
\end{aligned}
$$

The control inputs $u$ are given by

$$
\begin{aligned}
u_{\alpha^{\prime \prime}}= & -\left[g_{\alpha^{\prime \prime} \beta^{\prime \prime}} k_{\beta^{\prime}, \gamma^{\prime}}^{\beta^{\prime \prime}}\right. \\
& -g_{\alpha^{\prime \prime} \beta^{\prime \prime}} k_{\delta^{\prime}}^{\beta^{\prime \prime}} A^{\delta^{\prime} \alpha^{\prime}}\left(g_{\alpha^{\prime} \beta^{\prime}, \gamma^{\prime}}-g_{\beta^{\prime} \gamma^{\prime}, \alpha^{\prime}} / 2\right. \\
& \left.\left.-g_{\alpha^{\prime} \delta^{\prime \prime}} g^{\delta^{\prime \prime} \alpha^{\prime \prime}} g_{\beta^{\prime} \alpha^{\prime \prime}, \gamma^{\prime}}-g_{\alpha^{\prime} \delta^{\prime \prime}} k_{\beta^{\prime}, \gamma^{\prime}}^{\delta^{\prime \prime}}\right)\right] \dot{r}^{\beta^{\prime}} \dot{r}^{\prime} \gamma^{\prime} \\
& -g_{\alpha^{\prime \prime} \beta^{\prime \prime}} k_{\delta^{\prime}}^{\beta^{\prime \prime}} A^{\delta^{\prime} \alpha^{\prime}}\left[-\left(g_{\alpha^{\prime} \gamma^{\prime \prime}, \gamma^{\prime}}-g_{\gamma^{\prime} \gamma^{\prime \prime}, \alpha^{\prime}}\right.\right. \\
& \left.-g_{\alpha^{\prime} \delta^{\prime \prime}} g^{\delta^{\prime \prime} \beta^{\prime \prime}} g_{\gamma^{\prime \prime} \beta^{\prime \prime}, \gamma^{\prime}}\right) \dot{r}^{\prime} \dot{r}^{\prime \prime} \gamma^{\prime \prime} \\
& \left.+g_{\gamma^{\prime \prime} \beta^{\prime \prime}, \alpha^{\prime}} \dot{\gamma}^{\prime \prime} \dot{r}^{\beta^{\prime \prime}}+\nabla_{\alpha^{\prime}} U\right] .
\end{aligned}
$$

In the above,

$$
A_{\alpha^{\prime} \beta^{\prime}}=g_{\alpha^{\prime} \beta^{\prime}}+g_{\alpha^{\prime} \delta^{\prime \prime}} g_{\beta^{\prime} \alpha^{\prime \prime}}\left(\sigma^{\delta^{\prime \prime} \alpha^{\prime \prime}}-g^{\delta^{\prime \prime} \alpha^{\prime \prime}}\right)
$$

and $\dot{r}^{\beta^{\prime \prime}}$ can be eliminated, if desired, by making use of the relation

$$
\dot{r}^{\beta^{\prime \prime}}=g^{\alpha^{\prime \prime} \beta^{\prime \prime}} \widetilde{J}_{\alpha^{\prime \prime}}-\left(g^{\alpha^{\prime \prime} \beta^{\prime \prime}} g_{\alpha^{\prime} \alpha^{\prime \prime}}+k_{\alpha^{\prime}}^{\beta^{\prime \prime}}\right) \dot{r}^{\alpha^{\prime}} .
$$

In the last formula, $\widetilde{J}_{\alpha^{\prime \prime}}$ are constants.

Stability of the relative equilibria of the system associated with $\widetilde{\mathcal{R}}$ can be analyzed using the nonholonomic energy-momentum method (see [17]).

Remark. The equilibria and steady state motions of nonholonomic systems are not isolated. The above technique is used to stabilize the manifold of relative equilibria, but not a single relative equilibrium. For the latter goal, one needs a discontinuous feedback controller. However, it is possible to include dissipation emulating terms in the control input and achieve asymptotic stabilization of the manifold of steady state motions (see $[9,10]$ for details).

\section{Stabilization of the Unicycle with Rotor}

In this section we apply the technique developed above to the problem of stabilization of the slow vertical steady state-motions of the unicycle with rotor.

The Model. We now present the dynamical model of a homogeneous disk on a horizontal plane with a rotor. The rotor is free to rotate in the plane orthogonal to the disk. The rod connecting the centers of the disk and rotor keeps the direction of the radius of the disk through the contact point with the plane (i.e., the appropriate controller has already been implemented).

The configuration space for this system is $Q=S^{1} \times S^{1} \times$ $S^{1} \times S E(2)$, which we parameterize with coordinates $(\theta, \chi, \psi, \phi, x, y)$. As in Figure $1, \theta$ is the tilt of the unicycle itself, and $\psi$ and $\chi$ are the angular positions of the wheel of the unicycle and the rotor, respectively. The variables $(\phi, x, y)$, regarded as a point in $S E(2)$, represent the angular orientation and position of the point of contact of the wheel with the ground.

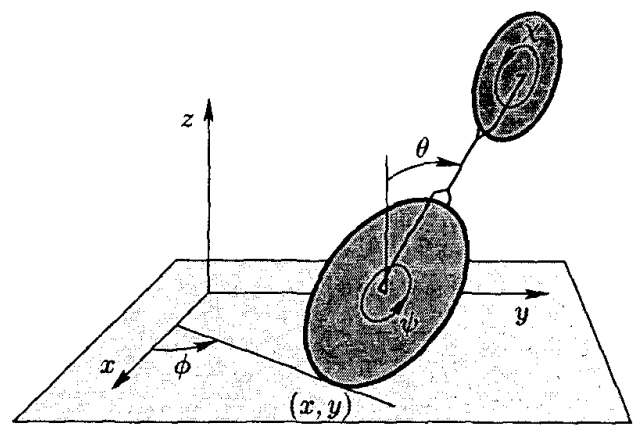

Figure 1: The configuration variables for the unicycle with rotor.

This mechanical system is $S O(2) \times S E(2)$-invariant; the groups $S O(2)$ represent the symmetry of the wheel, that is, the symmetry in the $\psi$ variable, while the group $S E(2)$ represents the Euclidean symmetry of the overall system. The action by the group element $(\alpha, \beta, a, b)$ on the configuration space is given by $(\theta, \chi, \psi, \phi, x, y) \mapsto(\theta, \chi, \psi+\alpha, \phi+\beta, x \cos \beta-y \sin \beta+$ $a, x \sin \beta+y \cos \beta+b)$.

System Parameters. We will use the following notations:

$M=$ the mass of the disk,

$R=$ the radius of the disk,

$A, B=$ the principal moments of inertia of the disk,

$\mathcal{A}, \mathcal{B}=$ the principal moments of inertia of the rotor,

$r=$ the rod length,

$\mu=$ the rotor mass.

Lagrangian and Constraints. The Lagrangian of this system has the standard form of kinetic minus potential energy:

$$
L=\dot{K_{\mathrm{d}}}+K_{\mathrm{r}}+\frac{M}{2} v_{M}^{2}+\frac{\mu}{2} v_{\mu}^{2}-V
$$

where,

$$
K_{\mathrm{d}}=\frac{1}{2}\left[A\left(\dot{\theta}^{2}+\dot{\phi}^{2} \cos ^{2} \theta\right)+B(\dot{\phi} \sin \theta+\dot{\psi})^{2}\right],
$$




$$
\begin{aligned}
K_{\mathrm{r}}= & \frac{1}{2}\left[\mathcal{A}\left(\dot{\phi}^{2} \sin ^{2} \theta\right)+\mathcal{B}(\dot{\chi}-\dot{\theta})^{2}\right], \\
v_{M}^{2}= & (\dot{x}-R \dot{\phi} \sin \theta \cos \phi)^{2}+(\dot{y}-R \dot{\phi} \sin \theta \sin \phi)^{2} \\
& +R^{2} \dot{\theta}^{2}+2 R \dot{\phi} \cos \theta(\dot{y} \cos \phi-\dot{x} \sin \phi), \\
v_{\mu}^{2}= & (\dot{x}-(R+r) \dot{\phi} \sin \theta \cos \phi)^{2} \\
& +(\dot{y}-(R+r) \dot{\phi} \sin \theta \sin \phi)^{2}+(R+r)^{2} \dot{\theta}^{2} \\
& +2(R+r) \dot{\phi} \cos \theta(\dot{y} \cos \phi-\dot{x} \sin \phi), \\
V= & M g R \cos \theta+\mu g(R+r) \cos \theta .
\end{aligned}
$$

The constraints are given by the standard conditions of rolling without slipping:

$$
\dot{x}=-\dot{\psi} R \cos \phi, \quad \dot{y}=-\dot{\psi} R \sin \phi .
$$

Constrained Lagrangian and Nonholonomic Momentum. The reduced Lagrangian for the unicycle with rotor is

$$
\begin{aligned}
L_{c}=\frac{1}{2}\left(\alpha \dot{\theta}^{2}\right. & +2 \beta \dot{\theta} \dot{\chi}+\beta \dot{\chi}^{2} \\
& \left.+I_{11}(\theta) \dot{\phi}^{2}+2 I_{12} \dot{\phi} \dot{\psi}+I_{22} \dot{\psi}^{2}\right)-V(\theta)
\end{aligned}
$$

where

$$
\alpha=A+M R^{2}+\mu(R+r)^{2}+\mathcal{B}, \quad \beta=\mathcal{B}
$$

are the components of the shape metric, and

$$
\begin{aligned}
& I_{11}=A \cos ^{2} \theta+\mathcal{A}+\left(B+M R^{2}+\mu R(R+r)\right) \sin ^{2} \theta, \\
& I_{12}=\left(B+M R^{2}+\mu R(R+r)\right) \sin \theta, \\
& I_{22}=B+M R^{2}+\mu R(R+r)
\end{aligned}
$$

are the components of the locked inertia tensor. The components of the nonholonomic momentum are

$$
\begin{aligned}
& p_{1}=\frac{\partial L_{c}}{\partial \dot{\phi}}=I_{11} \dot{\phi}+I_{12} \dot{\psi} \\
& p_{2}=\frac{\partial L_{c}}{\partial \dot{\psi}}=I_{12} \dot{\phi}+I_{22} \dot{\psi}
\end{aligned}
$$

For the unicycle with rotor, $p_{1}$ is the vertical (i.e., orthogonal to the $x y$-plane) component of the angular momentum of the system while $p_{2}$ is the component of the disk's angular momentum along the normal direction to the disk. See Zenkov, Bloch, and Marsden [17] for details concerning the nonholonomic momenta.

Reduced Equations. The reduced dynamics of the unicycle is governed by equations (1)-(3) with $r^{1}=\theta$, $r^{2}=\chi$, and the Routhian

$$
\mathcal{R}=\frac{1}{2}\left(\alpha \dot{\theta}^{2}+2 \beta \dot{\theta} \dot{\chi}+\beta \dot{\chi}^{2}-I^{a b}(\theta) p_{a} p_{b}\right)-V(\theta) .
$$

As usual, $I^{a b}$ are the components of the inverse inertia tensor.
The shape equations for $(\theta, \chi)$ describe the motion of the rod and rotor system, while the momentum equations for $\left(p_{1}, p_{2}\right)$ model the (coupled) wheel dynamics. The coefficients $\mathcal{D}_{a \alpha}^{b}$ in (3) for the unicycle with rotor are computed to be

$$
\begin{aligned}
& \mathcal{D}_{11}^{a}=I^{2 a}(M R+\mu(R+r)) R \cos \theta, \\
& \mathcal{D}_{21}^{a}=-I^{1 a}(M R+\mu(R+r)) R \cos \theta .
\end{aligned}
$$

See Zenkov, Bloch, and Marsden [17] for the details concerning how one derives and organizes such equations.

The slow vertical steady state motions of this system are represented by the relative equilibria

$$
\theta=0, \quad \dot{\chi}=0, \quad p_{1}=0, \quad p_{2}=p_{2}^{0} .
$$

Momentum Reduction and Stabilization. This system satisfies all conditions of section 2 . The momentum equations define an integrable distribution. The dynamics on the invariant manifolds $Q_{c}$ is governed by the equations

$$
\frac{d}{d t} \frac{\partial \mathcal{L}_{c}}{\partial \dot{\theta}}=\frac{\partial \mathcal{L}_{c}}{\partial \theta}, \quad \frac{d}{d t} \frac{\partial \mathcal{L}_{c}}{\partial \dot{\chi}}=u_{c},
$$

where

$$
\mathcal{L}_{c}=\frac{1}{2}\left(\alpha \dot{\theta}^{2}+2 \beta \dot{\theta} \dot{\chi}+\beta \dot{\chi}^{2}\right)-U_{\mathrm{c}}(\theta)
$$

and

$$
U_{c}(\theta)=\frac{1}{2} I^{a b}(\theta) \mathcal{P}_{c}(\theta, c), \mathcal{P}_{b}(\theta, c)+V(\theta)
$$

is the amended potential for the unicycle with rotor restricted to the invariant manifolds (5). Observe that the components of the shape metric for the unicycle with rotor are constants. We thus apply the approach of Bloch, Leonard, and Marsden $[4,5,7]$ and construct the controlled Lagrangians of the form

$$
\begin{aligned}
\widetilde{\mathcal{L}}_{c}=\frac{1}{2}\left(\alpha \dot{\theta}^{2}+2 \beta \dot{\theta}(\dot{\chi}+k \dot{\theta})\right. & \left.+\beta(\dot{\chi}+k \dot{\theta})^{2}\right) \\
& +\frac{\sigma}{2}(k \dot{\theta})^{2}-U_{c}(\theta)
\end{aligned}
$$

where $k$ and $\sigma$ are constants. The uncontrolled dynamics associated with $\widetilde{\mathcal{L}}_{c}$,

$$
\frac{d}{d t} \frac{\partial \widetilde{\mathcal{L}}_{c}}{\partial \dot{\theta}}=\frac{\partial \widetilde{\mathcal{L}}_{\mathrm{c}}}{\partial \theta}, \quad \frac{d}{d t} \frac{\partial \widetilde{\mathcal{L}}_{c}}{\partial \dot{\chi}}=0,
$$

is then forced to be equivalent to the controlled dynamics (15). This requirement implies $\sigma=-\beta / k$ and defines the control laws by

$$
u_{c}=\frac{k \beta}{\alpha-\beta-k \beta} \frac{\partial U_{c}}{\partial \theta} .
$$


These control laws act on the systems on appropriate invariant manifolds (5). See [4-7] for details concerning the matching process and identification of the control inputs.

Using the approach of section 3 , we obtain the controlled Routhian for the unicycle with rotor:

$$
\begin{aligned}
\widetilde{\mathcal{R}}=\frac{1}{2}\left(\alpha \dot{\theta}^{2}+2 \beta \dot{\theta}(\dot{\chi}\right. & \left.+k \dot{\theta})+\beta(\dot{\chi}+k \dot{\theta})^{2}\right) \\
& +\frac{\sigma}{2}(k \dot{\theta})^{2}-\frac{1}{2} I^{a b} p_{a} p_{b}-V(\theta) .
\end{aligned}
$$

The control law rewritten in the terms of the amended potential $U(\theta, p)$ becomes:

$$
u=\frac{k \beta}{\alpha-\beta-k \beta} \nabla_{\theta} U(\theta, p) .
$$

Recall that $p$ in the amended potential is a dynamic variable and not a function of $r$. We emphasize that the derivative $\nabla_{\theta} U$ can be evaluated explicitly:

$$
\nabla_{\theta} U(\theta, p)=\frac{\partial\left(I^{a b} p_{a} p_{b} / 2+V(\theta)\right)}{\partial \theta}+\mathcal{D}_{a \theta}^{c} I^{a b} p_{b} p_{c}
$$

We can conclude stability of the relative equilibria $\theta=$ $0, p_{1}=0, p_{2}=p_{2}^{0}$ using the nonholonomic energymomentum method applied to the controlled Routhian. The steady state motions under consideration become stable if one chooses

$$
k>\frac{\alpha-\beta}{\beta^{2}} .
$$

\section{Conclusions}

The proposed method allows one to establish explicitly the stabilizing control inputs for a class of underactuated nonholonomic systems. We intend in a future publication to relax some of the assumptions on the Lagrangian and constraints made in this paper.

\section{References}

[1] Auckly, D., L. Kapitanski, \& W. White, Control of Nonlinear Underactuated Systems, Commun. Pure Appl. Math. 53, 2000, 354-369.

[2] Blankenstein, G., R. Ortega \& A. van der Schaft, The Matching Conditions of Controlled Lagrangians and Interconnection and Damping Assignment Passivity Based Control, 2001, (preprint).

[3] Bloch, A.M., P.S. Krishnaprasad, J.E. Marsden, \& R. Murray, Nonholonomic Mechanical Systems with Symmetry, Arch. Rat. Mech. An. 136, 1996, 21-99.

[4] Bloch, A.M., N. Leonard, \& J.E. Marsden, Stabilization of Mechanical Systems Using Controlled Lagrangians, Proc. CDC 36, 1997, 2356-2361.
[5] Bloch, A.M., N. Leonard, \& J.E. Marsden, Matching and Stabilization by the Method of Controlled Lagrangians, Proc. CDC 37, 1998, 1446-1451.

[6] Bloch, A.M., N. Leonard, \& J.E. Marsden, Potential Shaping and the Method of Controlled Lagrangians, Proc. CDC 38, 1999, 1652-1657.

[7] Bloch, A.M., N.E. Leonard, \& J.E. Marsden, Controlled Lagrangians and the Stabilization of $\mathrm{Me}-$ chanical Systems I: The First Matching Theorem, IEEE Trans. on Systems and Control 45, 2000, 22532270.

[8] Bloch, A.M., D-E. Chang, N.E. Leonard, \& J.E. Marsden, Controlled Lagrangians and the Stabilization of Mechanical Systems II: Potential Shaping, Trans. IEEE on Autom. Contr. 46, 2001, 1556-1571.

[9] Bloch, A.M., M. Reyhanoglu, \& N.H. McClamroch, Control and Stabilization of Nonholonomic Dynamic Systems, IEEE Trans. Autom. Contr. 37, 1992, 1746-1757.

[10] Brockett, R. W. Asymptotic Stability and Feedback Stabilization. Differential Geometric Control Theory, Birkauser, Boston, 1983, 181-191.

[11] Hamberg, J., General Matching Conditions in the Theory of Controlled Lagrangians, Proc. $C D C \mathbf{3 8}$, 1999, 2519-2523.

[12] Hamberg, J,. Controlled Lagrangians, Symmetries and Conditions for Strong Matching. In: Lagrangian and Hamiltonian Methods for Non-linear Control, Elsevier, 2000.

[13] Marsden, J.E., Lectures on Mechanics, London Mathematical Society Lecture Note Series 174, Cambridge University Press, 1992.

[14] Maschke, B., R. Ortega \& A. van der Schaft, Energy-Based Lyapunov Functions for Forced Hamiltonian Systems with Dissipation, IEEE Trans. Automat. Control, 45, 2001,1498-1502.

[15] Ortega, R., A. Loria, P. J. Nicklasson \& H. SiraRamirez, Passivity-based Control of Euler-Lagrange Systems, Springer-Verlag, Communication \& Control Engineering Series, 1998.

[16] Ortega, R., M. W. Spong, F. Gómez-Estern \& G. Blankenstein, Stabilization of Underactuated Mechanical Systems via Interconnection and Damping Assignment, IEEE Trans. Aut. Control (submitted), 2001.

[17] Zenkov, D.V., A.M. Bloch, \& J.E. Marsden, The Energy-Momentum Method for Stability of Nonholonomic Systems, Dynamics and Stability of Systems 13, 1998, 123-165.

[18] Zenkov, D.V., A.M. Bloch, N.E. Leonard, \& J.E. Marsden Matching and Stabilization of LowDimensional Nonholonomic Systems. Proc. CDC 39, 2000, 1289-1295. 